\title{
An Image Enhancement Algorithm Based on Second Generation
}

\section{Wavelet Integer Transform}

\author{
Yi Zhang ${ }^{1, \text { a }}$, Haifeng Wang ${ }^{1}$ \\ ${ }^{1}$ Information Center, JiangSu University of Technology, Changzhou, JiangSu, China \\ alubianinfo@foxmail.com
}

\begin{abstract}
Keywords: lifting wavelets; second generation wavelet; homomorphic filtering; histogram equalization
\end{abstract}

\begin{abstract}
Traditional histogram enhancement algorithm to enhance image has the defect of over-bright image, amplified noise, and missing image details. In view of these defects, this paper proposes an image enhancement algorithm based on second generation wavelet integer transform. At first, use the second generation wavelet integer transform to decompose the original image with wavelet. Then, integer low-frequency subimage $c a$ in the wavelet domain is calculated with histogram equalization to figure out low-frequency subimage $c a^{\prime}$. And make an evenly spaced arrangement for integer low-frequency subimage ca' of equalized histogram between the maximum and the minimum to figure out new low-frequency subimage $c a^{\prime \prime}$. At last, low-frequency subimage $c a^{\prime \prime}$ is reconstructed. In comparison with classic histogram equalization algorithm and experimental results of homomorphic filtering, algorithm in this paper keeps the optimal in enhancing image, suppressing noise and preserving brightness.
\end{abstract}

\section{Introduction}

Image enhancement is to improve the visual effects of images and make them more clear and intuitive and make it appropriate to analysis. There are many algorithms enhancing the images. The histogram equalization is much more classic and effective. Despite the obvious advantages of fast arithmetic speed and great enhancement effectiveness, it still has the following shortcomings: (1) The histogram equalization would cause poor layering sense because of the small dynamic range of the original image's gray scale, its poor quality and uneven histogram equalization. (2) After the histogram equalization, the noise in the original is obviously enlarged. (3) If the image gray scale is close to 0 , during the histogram equalization, a brighter diluted image would be output when the very narrow range of dark pixels is mapped to the output image. This would affect the basic features of the image (for instance, the average luminance would be changed; the details would be lost), which would finally influence the visual effects to enhance the image and make the applications of histogram algorithm limited ${ }^{[1-3]}$.

\section{Several classic methods to enhance image}

Histogram Equalization Enhancement. The basic idea of histogram equalization is to transform the unbalanced histogram of the original image to form of uniform distribution. Namely, convert the input image to have the same pixel points in each gray level (The histogram output is smooth and distribution is uniform). This makes dynamic range of the image pixels wider and contrast stretched effectively so as to achieve the effect of image enhancement. Histogram 
equalization enhancement has a disadvantage that if the noise image is enhanced, a little noise will be greatly enlarged at the same time. The following is a digital image histogram equalization algorithm formula.

$$
p_{r}\left(r_{k}\right)=n_{k} / n \quad\left(0 \leq r_{k} \leq 1 \quad \mathrm{k}=0,1,2, \ldots, \mathrm{L}-1\right)
$$

In formula (1), $\mathrm{L}$ is the sum of gray levels. $p_{r}\left(r_{k}\right)$ is probability of Class $\mathrm{K}$ gray value. $n_{k}$ is the times of Class $\mathrm{K}$ gray value existed in the image. ${ }^{n}$ is sum of all pixels in the image.

New image grey value is as follows:

$$
s_{k}=T\left(r_{k}\right)=\sum_{j=0}^{k} n_{j} / n=\sum_{j=0}^{k} p_{r}\left(r_{j}\right) \quad\left(\quad 0 \leq r_{j} \leq 1 \quad \mathrm{k}=0,1,2, \ldots, \mathrm{L}-1 \quad\right)
$$

Based on formula (2), each pixel in digital image is counted to figure out new gray value. To use new gray value to replace original gray value, that is the traditional histogram equalization processing ${ }^{[4]}$.

Homomorphic Filtering Enhancement. The basic idea of homomorphic filtering is to transform a linear problem into a nonlinear problem. Assuming that the image $f(x, y)$ can be expressed by multiplication of lighting function $I(x, y)$ and reflective function $r(x, y)$, that is, $f(x, y)$ $=\mathrm{i}(\mathrm{x}, \mathrm{y}) \mathrm{r}(\mathrm{x}, \mathrm{y})$. Take the logarithm of image $\mathrm{f}$, add the image brightness to reflective function, and then take the Fourier transform. After that, act homomorphic filtering function $\mathrm{H}(\mathrm{u}, \mathrm{v})$ on brightness after transformation and reflective function respectively, and then take inverse operation respectively. The process is shown in figure 1.

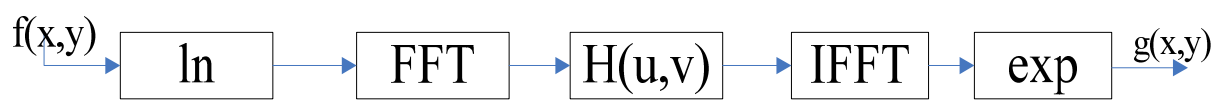

Figure 1 Flow chart of the homomorphic filtering

Homomorphic filtering chooses $H_{L}<1, H_{H}>1$. Filter function reduces low frequency (brightness) and increases high frequency (reflection). As a result, image dynamic range compression and contrast are enhanced ${ }^{[5-6]}$.

\section{The Second Generation Wavelet Integer Transform Equalization Enhancement}

Lifting Wavelets. In the process of wavelet transform, the traditional wavelet transform filter output is a floating point number, but pixel values of image are integers. Wavelet lifting proposes a new point of view on wavelet structure, namely, the wavelet lifting scheme, which is also known as the second generation wavelet transform. Wavelet lifting really has reversibility, so the boundary effect needn't considering. Compared with the traditional wavelet transform, lifting scheme mainly has the following advantages: a) It inherits multi-resolution property of the first generation wavelet. Image reconstruction quality doesn't restrict the length of input sequence, and has the ability of transformation on image of any sizes; b) Construction of wavelets is entirely carried out within the airspace, with no need for Fourier analysis theory; c) Tools used is fairly simple, mainly the Euclidean division of Laurent series. All the traditional wavelet can be decomposed by basic lifting and dual of the lifting scheme; d) It has high speed of calculation and save storage space; e) Integer-to-integer wavelet transform can be realized so as to achieve wavelet-based lossless compression.

Decomposition process of lifting wavelet can be divided into 3 steps, namely, split, predict and update: 
Step1. Split: The original input signal is decomposed into the even and odd number sequences. Decomposition process is expressed as $F\left(s_{j}\right)=\left(s_{j-1}, d_{j-1}\right)$; Thereinto, $s_{j-1}$ represents low frequency approximation components, $d_{j-1}$ represents signal's high frequency detail component, and $F(s, j)$ represents decomposition process.

Step2. Predict: Make use of the relativities of data to predict the odd number sequence with an even number sequence. Prediction error is $d_{j-1}=d_{j-1}-P\left(s_{j-1}\right)$; Prediction operator is $P[\bullet]$.

Step3. Update: Create a better data set $s_{j-1}$ by operator $U$ to keep some features of the original data set $s_{j}$. The expression of the update process is $s_{j-1}=s_{j-1}+U\left(d_{j-1}\right)$; Update operator is $U[\bullet]$.

Because wavelet lifting of image is completely reversible process, and its inverse transform and the forward transform are structurally symmetrical and implements opposite operation, which accurate reconstruction can be realized.

Subimage Wavelet Coefficients Equalization Algorithm. The traditional discrete wavelet transform based on convolution has massive and complex computation and demands high on storage space, interfering with its hardware implement. However, lifting wavelet algorithm has properties of simple structure, low calculating amount, saving storage space, and reversible integer-to-integer transform, which is convenient for hardware implement ${ }^{[9-11]}$.

The original image is decomposed by using lifting wavelet integer transform to get an integer low frequency subimage $c a$ and triple high frequency subimage decomposition coefficients;

To get the sum of integer low frequency subimage $c a$ 's coefficients $k$, namely, $n(k)$;

To calculate coefficient's maximum value $K_{\max }$ and minimum value $K_{\min }$ of integer low frequency subimage $c a$;

Integer low frequency subimage coefficient $\mathrm{k}$ and count $\mathrm{n}(\mathrm{k})$ sum cumulatively $c d f(k)=\sum_{i=1}^{k} n(k) 0 \leq k \leq K_{\max }(3)$

To calculate the new coefficient of integer low frequency subimage $c a$ equalization $g(k)=K_{\min }+\frac{c d f(k) \times\left(K_{\max }-K_{\min }\right)}{m \times n} K_{\min } \leq k \leq K_{\max }$ (M and $\mathrm{n}$ are the line numbers and column numbers of image respectively). Rounding-off method is used to take the whole to constitute new low frequency subimage $\mathrm{ca}^{\prime}(4)$;

To calculate the histogram and coefficients series of $\mathrm{ca}^{\prime}$;

To calculate maximum value $\mathrm{M}$ and minimum value $\mathrm{N}$ of $\mathrm{ca}^{\prime}$. Make an evenly spaced equalization in the range of $[\mathrm{M} \mathrm{N}]$ and calculate new coefficient of new low frequency subimage $\mathrm{ca}^{\prime \prime}$;

To reconstruct new image of low frequency subimage $c a^{\prime \prime}$ directly.

Evaluation of Image Enhancement and De-noising Performance. To examine algorithm's 
ability in image enhancement and de-noising, choose the mean square error (MSE), average brightness difference $(\Delta Y)$, and contrast increment to evaluate objectively algorithm in this paper [12-14].

MSE: $M S E=\frac{1}{m \times n} \sum_{i=1}^{m} \sum_{j=1}^{n}[f(i, j)-\bar{f}(i, j)]^{2}$

Average brightness difference: $\Delta Y=Y_{F}-Y_{f}=\sum_{k_{F}=0}^{k_{F}=255} k_{F} p\left(k_{F}\right)-\sum_{k_{f}=0}^{k_{f}=255} k_{f} p\left(k_{f}\right)$

Contrast increment: $\Delta=\frac{C^{\prime} \bar{f}}{C_{f}}$

In the formula $(5), f(i, j)$ is the noise of original, $\bar{f}(i, j)$ is de-noising image, and $\mathrm{M}$ and $\mathrm{N}$ represent the line numbers and column numbers of image respectively; if MSE is smaller, de-noising effect is better.

In the formula (6), $Y_{F}$ is average brightness of original image, $Y_{f}$ is image enhanced, $\Delta Y$ is difference value. If the difference value is smaller, brightness of the enhanced image gets closer to the original image, which indicates enhanced algorithm keeps better image brightness, conversely poorer image brightness.

Contrast increment is the ratio of the local contrast of original image contrast and enhanced image. With $3 \times 3$ sliding windows, the local contrast of each window is calculated according to $\left(x_{\max }-x_{\min }\right) /\left(x_{\max }+x_{\min }\right)$, and then average value is taken ${ }^{[15]}$. In the formula $(7), C^{\prime} \bar{f}$ is the mean ratio of the local contrast of enhanced image. $C_{f}$ is the mean ratio of the local contrast of original image. The greater the contrast increment is, the better effect of enhancement is.

\section{Results and Analysis}

Based on the MATLAB for experimental study, this paper writes programs for the three kinds of processing algorithm above to carry on experimental contrast. Experimental subjects are standard Lena image and Gaussian noise Lena image of 0.05 variance.

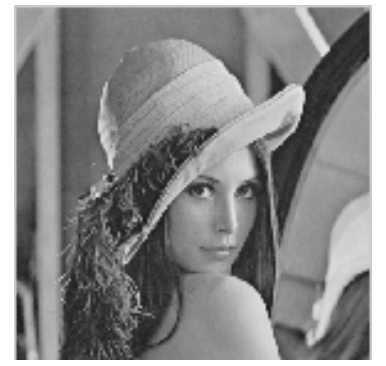

(a) Original Image

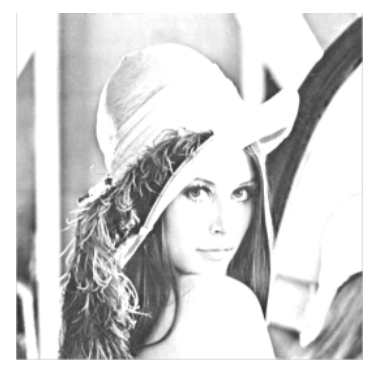

(b) Homomorphic Filtering Enhancement 


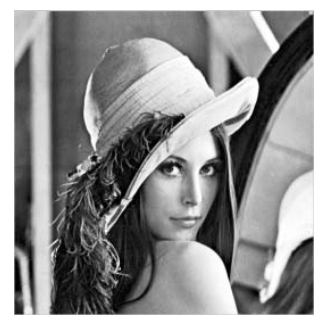

(c) Histogram Equalization Enhancement

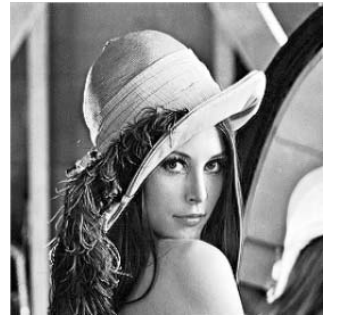

(d) Algorithm in this paper

Figure 2 Experimental results of the original image

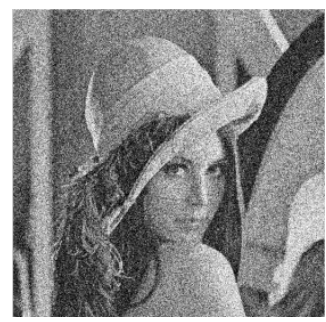

(a) Noised Image

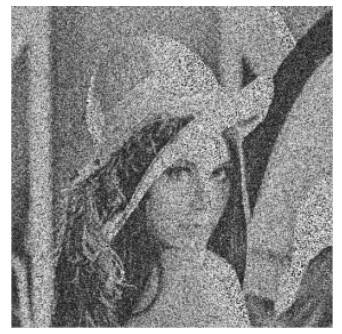

(c) Histogram Equalization Enhancement

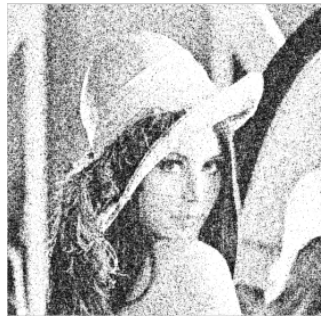

(b) Homomorphic Filtering Enhancement

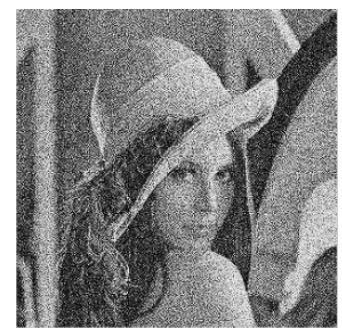

(d) Algorithm in this Paper

Figure 3 Experimental results of the noised image

The following are original Lena image and histograms after enhanced original image by three kinds of algorithms:

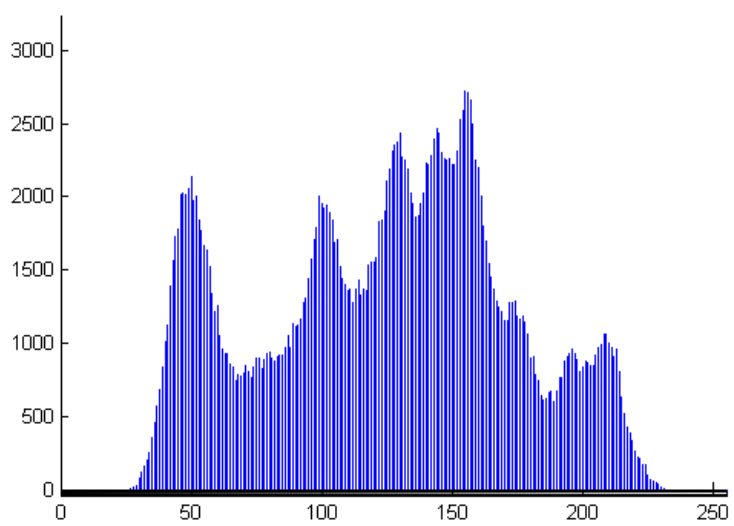

(a)Original Lena Image

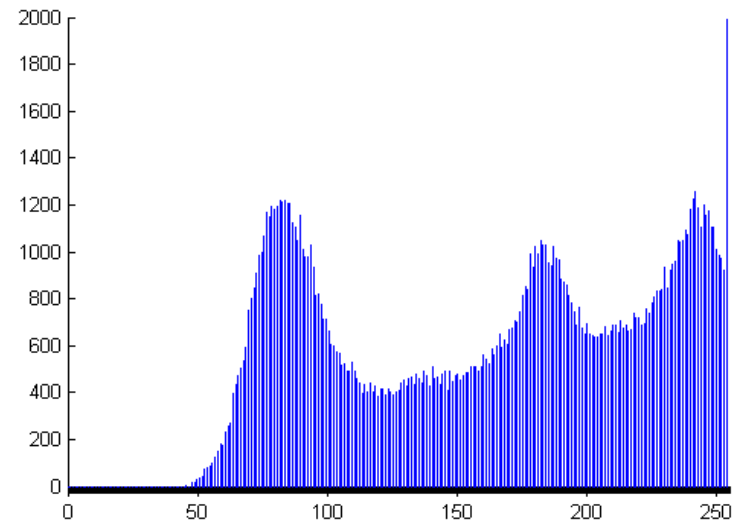

(b) Homomorphic Filtering Enhancement Image 


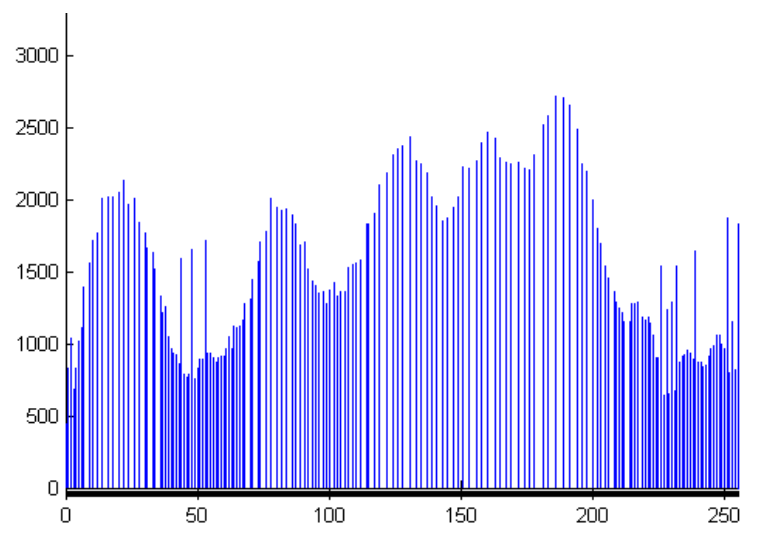

(c)Histogram Equalization Enhancement Image

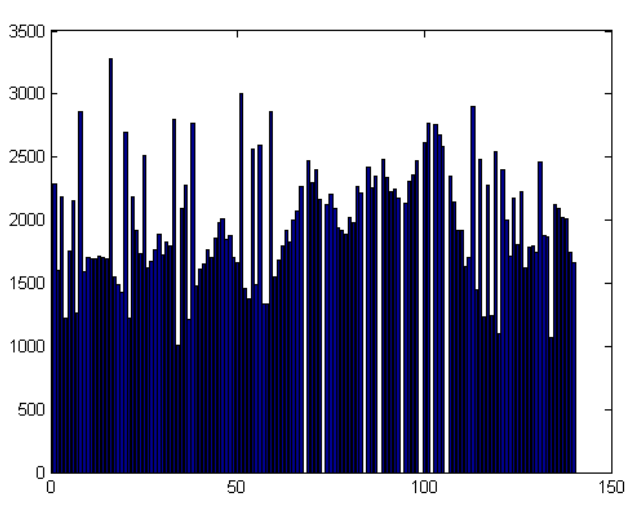

(d) Enhancement Image by Algorithm in this Paper

Figure 4 Histogram

Notes: Figure 2a is original image. Figure 3a is original image with Gaussian noise of 0.05 variance. Three kinds of enhancement algorithm all process noised image in experiment. Figure $2 b$ and Figure $3 b$ are the enhancement results of homomorphic filtering enhancement coefficients taken as $H_{H}=2.0$ and $H_{L}=0.5$, and sharpening coefficient taken as $\mathrm{c}=1.1$.

In Figure 2, Lena image has been enhanced by three kinds of algorithms. It can be seen from enhancement effect that enhanced image by histogram equalization and algorithm in this paper is difficult to be visually distinguished from pros and cons. Both enhancement effects are significantly better than that of homomorphic filtering, and image level is also clearer. The corresponding histogram Figure $4 c$ Figure $4 d$ contrast dynamic range are wide, while the enhanced image by homomorphic filtering is overall brighter a tad bit and not clear, and loses detail as well. Histogram Figure $4 \mathrm{~b}$ also indicates that the image grayscale is concentrated in the highlight area. Contrast range is relatively narrow. There is almost no low gray value.

Figure 3 is enhanced Lena image with Gaussian noise of 0.05 variance. Algorithm in this paper works best with clear image, good contrast and noise suppression among the three kinds of algorithms. Although histogram equalization algorithm enhances image, noise is be amplified and contrast is poor at the same time. As for noise suppression, suppression effect of homomorphic filtering algorithm is similar to that of algorithm in this paper.

Enhancement effect of three kinds of algorithms is analyzed qualitatively from the point of view of subjectivity. Next, three performance indexes that evaluates image de-noising and enhancement effect are used to carry on quantitative analysis of noise image's de-noising and enhancement effect of the three enhancement methods. pixel gray value changes after image enhancement, so noised enhancement image and de-noising enhancement image of original image are compared to calculate the three performance indexes respectively. The calculation results are shown in Table 1.

Table.1 Results of the computation

\begin{tabular}{llll}
\hline Performance index & $\begin{array}{l}\text { Homomorphic } \\
\text { filtering }\end{array}$ & $\begin{array}{l}\text { Histogram } \\
\text { equalization }\end{array}$ & $\begin{array}{l}\text { Algorithm in this } \\
\text { paper }\end{array}$ \\
\hline MSE & 391.41 & 458.86 & 255.71 \\
\hline $\begin{array}{l}\text { Average brightness } \\
\text { difference }\end{array}$ & 111.31 & 34.17 & 53.93 \\
\hline Contrast increment & 0.91 & 2.09 & 2.29 \\
\hline
\end{tabular}


In Table 1, with regard to contrast increment, the algorithm in this paper is greater than histogram equilibrium and histogram equilibrium is greater than homomorphic filtering, which suggests the enhancement effect of algorithm in this paper of the three is the best, followed by the histogram equalization and finally homomorphic filtering. In table 1, with regard to MSE, the algorithm in this paper is smaller than homomorphic filtering and homomorphic filtering is smaller than histogram equilibrium, that is, noise suppression of algorithm in this paper is optimal. As for average brightness difference, histogram equilibrium is smaller than algorithm in this paper and algorithm in this paper is smaller than homomorphic filtering. Based on the subjective and objective analysis above, image enhancement and noise suppression of algorithm in this paper are optimal, while brightness preservation of original image, is not as good as that of histogram equalization.

\section{Conclusions}

In view of the low contrast and the enhancement processing of images with noises, this paper proposes an image enhancement algorithm based on second generation wavelet integer transform. Compared to the classical histogram equalization of airspace and the homomorphic filtering in the domain of frequency, the algorithm presented in this paper can greatly contribute to improve the image contrast and suppress its noises.

\section{Acknowledgements}

This work is supported by Youth Science Foundation of Jiangsu University of Technology (KYY14017; KYY13033).

\section{References}

[1] Fisee Technology. Wavelet Analysis Theory and MATLAB7 Application [M]. Beijing: Publishing House of Electronics Industry, 2005.

[2] SU Zhi-yuan \& ZHOU Xiao-guang, et al. Infrared Images Enhancement Based-on Balanced Spacing Density of Gray-Scale Equalization [J]. JOURNAL OF JILIN UNIVERSITY (INFORMATION SCIENCE EDITION), 2010, 28 (6): 592-595.

[3] SONG Yan-feng, SHAO Xiaopeng \& XU Jun. New enhancement algorithm for infrared image based on double plateaus histogram [J]. INFRARED AND LASER ENGINEERING, 2008, 37 (2): 308-311.

[4] YANG Yan-chun, DANG Jian-wu \& WANG Yang-ping. A Medical Image Fusion Method Based on Lifting Wavelet Transform and Adaptive PCNN [J]. Journal of Computer-Aided Design \& Computer Graphics, 2012, 24(4): 494-499.

[5] LIN De-gui. Lifting Wavelet Image De-noising Based on Edge Detection [J]. Journal of Changchun University, 2011, 21 (8): 31-33.

[6] LI Guang-qiong, YU Shao-qian \& CHEN Rong-yuan. Image Enhancement with Adaptive Lifting Wavelet Framework Based on Texture [J]. Computer Applications and Software, 2014, 31(3): 191-194.

[7] LI Jin-lun, CUI Shaohui \& WANG Ming. Threshold de-noising method for mixed noise based on improved median filter and lifting wavelet transform [J]. Journal of Applied Optics, 2014, 35 (5): 817-822. 
[8] WANG Hai-feng. A Histogram Enhancement Algorithm about Multilateral Wavelet Coefficients on Wavelet Domain [J]. Journal of Shanxi University (Natural Science Edition), 2013, 36 (3): 344-349.

[9] XU Tao \& LI Guanzhang. ADAPTIVE DETAIL ENHANCEMENT ALGORITHM OF COLOUR IMAGE BASED ON WAVELET TRANSFORM [J]. COMPUTER APPLICATIONS AND SOFTWARE, 2011, 28 (3): 240-242

[10] LIU Bin \& JIN Wei-qi, et al. Infrared Image Detail Enhancement Based on the Spatial and Frequency Domain Processing [J]. Infrared Technology, 2011, 33 (8): 477-482.

[11] YANG Jia-hong \& XIANG Jin, et al. Novel algorithm of detail enhancement for IR image [J]. Laser \& Infrared, 2012, 42 (5): 579-583.

[12] Chen S D, Ramli A R. Contrast enhancement using recursive mean-separate histogram equalization for scalable brightness preservation [J]. IEEE Transactions on Consumer Electronics, 2003, 49 (4):1301-1309.

[13] Sim K S, Tso C P, Tan Y Y. Recursive sub2image histogram equalization applied to gray scale images [J]. Pattern Recognition Letters, 2007, 28 (10): 1209-1221.

[14] Yeganeh H, Ziaei A, Rezaie A. A novel approach for contrast enhancement based on histogram equalization [C] // Computer and Communication Engineering, International Conference on. Kuala Lumpur: IEEE Communications Society ,2008: 256-260.

[15] WANG Hai-feng, WU Dong-yan \& ZHANG Yi. Image Enhancement Algorithm of Wavelet Coefficients Equalization about Wavelet Transform [J]. Video Engineering, 2011, 35(19): 27-29. 